\title{
Civilisations
}

Revue internationale d'anthropologie et de sciences

humaines

66 | 2017

L'alcool rituel et les ethnographes

\section{Les ancêtres aiment boire du rhum !}

(In)visible ivresse et questions réflexives sur le boire rituel

\section{Delphine Burguet}

\section{(2) OpenEdition}

1 Journals

Édition électronique

URL : http://journals.openedition.org/civilisations/4448

DOI : $10.4000 /$ civilisations.4448

ISSN : 2032-0442

Éditeur

Institut de sociologie de l'Université Libre de Bruxelles

Édition imprimée

Date de publication : 31 août 2017

Pagination : 123-138

ISBN : 978-2-9602017-1-0

ISSN : 0009-8140

\section{Référence électronique}

Delphine Burguet, «Les ancêtres aiment boire du rhum ! », Civilisations [En ligne], 66 | 2017, mis en ligne le 31 août 2020, consulté le 25 février 2021. URL : http://journals.openedition.org/civilisations/ 4448 ; DOI : https://doi.org/10.4000/civilisations.4448

(c) Tous droits réservés 


\title{
Les ancêtres aiment boire du rhum ! (In)visible ivresse et questions réflexives sur le boire rituel
}

\author{
Delphine BURGUET
}

Résumé : A Madagascar, les rituels magico-religieux orchestrés par les devins-guérisseurs sont mis en scène dans le respect des codes cultuels. Certaines substances sont considérées comme nécessaires au bon déroulement et au caractère efficace de l'action : parmi elles, le rhum, qui est utilisé à la fois en libation et en boisson. Ainsi, sa consommation par les maîtres rituels ou les médiums s'inscrit dans une pratique sociale légitimée, à caractère positif car elle valorise la puissance et la maîtrise dans un état alcoolisé. Parmi ces maîtres, ceux qui boivent de l'alcool sans montrer un état d'ivresse sont considérés comme des spécialistes confirmés. Néanmoins, le terrain ethnographique qui articule observations, entretiens et prises de vue photographiques et filmiques amène à interroger l'ivresse qui s'ensuit de la consommation de rhum. La question traitée est celle d'une relecture et d'un décentrement du regard ethnologique sur les pratiques $d u$ boire. Alors que l'ivresse semble mesurée et de fait indécelable dans l'action rituelle, elle est repérable dans un contexte distancié et éloigné de l'expérience du terrain ethnographique. Cette réflexivité travaille l'idée d'une hiérarchisation des données qui influence la production de l'analyse ethnologique.

Mots-clé : Madagascar, rhum, transe de possession, ivresse, anthropologie visuelle, réflexivité.

Abstract: In Madagascar, the magical and religious rituals orchestrated by the diviners-healers are performed in accordance with cultural standards. Some substances are considered necessary for the smooth functioning and efficacy of the action: among them, rum is used both as libation and as drink. Its consumption by ritual masters or mediums is hence part of a legitimate social practice because it values power and control in an alcoholic state. Among those masters, those who drink alcohol without showing a state of intoxication are considered to be specialists. Nevertheless, the ethnographic field that articulates observations, interviews and photographic and filmic shots, leads to question the drunkenness after the consumption of rum. The issue of this article is about a re-reading and a decentering of the ethnological viewpoint on the practices of drinking. While drunkenness seems measured and undetectable in ritual action, it can be spotted in a distant context, far from the experience of the ethnographic fieldwork. These re-reading questions the idea of a hierarchy of data that influences the production of ethnological analyze.

Keywords: Madagascar, rum, spirit possession, drunkenness, visual anthropology, reflexivity. 


\section{Introduction}

Dans un contexte religieux et sur un terrain ethnographique situé sur les Hautes Terres centrales de Madagascar, l'article propose tout d'abord d'explorer les différents usages du rhum dans un rapport de médiation avec les ancêtres afin d'appréhender l'environnement social dans lequel l'analyse réflexive se construit. Les rituels magicoreligieux orchestrés par les devins-guérisseurs sont mis en scène dans le respect des codes cultuels. Ainsi, certaines substances et objets sont considérés comme nécessaires au bon déroulement et au caractère efficace de l'action : parmi eux, l'alcool et plus précisément le rhum, est utilisé à la fois en libation et en boisson. Son absence priverait le rituel de sens.

Sa consommation s'inscrit dans une pratique sociale légitimée, à caractère positif car elle valorise la puissance et la maîtrise de soi dans un état alcoolisé. Selon les maîtres religieux, ceux qui boivent de l'alcool sans montrer un état d'ivresse sont considérés comme des spécialistes confirmés car ils maîtrisent la transe de possession. Dans le discours émique, ce ne sont pas eux qui boivent mais les ancêtres qui les investissent. L'authenticité de la transe est ainsi démontrée par la mise en scène d'une consommation sans ivresse apparente. Il devient clair que ce n'est pas le possédé qui boit, c'est l'esprit lui-même ${ }^{1}$.

L'article engage un questionnement sur le boire rituel qui enclenche un cadre de socialisation propre et indépendant du contexte ordinaire, où l'acte de boire devient fondamental. Il montre les possibles usages du rhum, les codes rituels associés, l'idée de commensalité et de médiation entre des ancêtres et des vivants ainsi que l'acte performant d'un corps enivré qui met en scène des esprits buveurs.

Il propose aussi une lecture réflexive, un retour sur l'expérience du terrain vécue par l'ethnologue et sur les pratiques rituelles alcoolisées qui ont été observées. Le film ethnographique permet la construction d'une réalité distanciée : l'ivresse est rendue visible. Alors qu'elle semble indécelable durant l'action rituelle, elle est repérable dans le temps de l'analyse. Par ailleurs, cette relecture questionne l'autocensure chez l'ethnologue (refuse-t-on de voir la défaillance ?) et le sentiment de trahison vis-à-vis des personnes étudiées qui peut résulter des expériences ethnographiques. Elle revisite ainsi des matériaux d'enquête jusque-là écartés de la recherche : dans la hiérarchisation des données, il y a celles que l'on range et celles qui nous dérangent.

\section{Ritualiser le rhum}

Tout comme les Antilles et les autres îles de l'océan Indien occidental (Réunion et Maurice), Madagascar produit et exporte du rhum dès le début de la période coloniale, mais il joue un rôle mineur dans l'économie de l'exportation, les trois principales îles françaises productrices étant la Martinique, la Guadeloupe et la Réunion (Huetz de

1 Ce type de discours qui valorise l'esprit comme l'acteur scénique de la consommation d'alcool est une question centrale ailleurs. Lambek mentionne lui aussi que celui qui boit est l'esprit, non celui qui est possédé. Ainsi, à Mayotte, les possédés peuvent être de bons musulmans car ce ne sont pas eux qui boivent du rhum (1981). Toutefois, retenons une situation inverse sur un terrain malgache : Harnik mentionne que "It's not the spirits who are talking, just the alcohol is talking (barisa fo miteny)" (1991 : 44). 
Lemps 1997 ; Fahrasme et Ganou-Parfait 1997). A Madagascar, au niveau local, son usage s'est répandu en partie par l'intermédiaire des colons, notamment créoles, qui l'utilisent dans les échanges commerciaux avec les populations locales. Alors que le rhum devient un produit marchand et d'échange recouvrant des enjeux économiques pour la Grande île, les pratiques d'alcoolisation par les populations indigènes sont estimées comme un problème sérieux par les médecins coloniaux. La question de la lutte contre l'alcoolisme est ainsi une priorité des politiques de santé publique et de l'assistance médicale indigène. Parallèlement aux mesures d'hygiène prises pour enrayer les maladies contagieuses, l'alcoolisme entre, de fait, dans les programmes sanitaires (Burguet 2014).

C'est dans ce contexte politique que s'installe progressivement l'usage normé du rhum dans l'espace domestique et dans celui dédié aux rituels ancestraux, remplaçant progressivement les anciennes boissons, notamment l'hydromel. Ce changement des liquides alcoolisées dans le domaine religieux correspond aux manifestations de plus en plus dominantes des possessions par des esprits royaux et princiers, qui durant la colonisation et la décolonisation montraient leurs désaccords politiques par la médiation ancestrale. Les possédés, intermédiaires entre les esprits et les vivants, manifestaient leur présence et revendiquaient une identité locale, autochtone, parfois anti-chrétienne. Si le rhum a pu faire figure de contre-pouvoir durant une période de contestation à présent clôturée, il a aujourd'hui trouvé sa place dans les milieux sociaux ordinaires et dans ceux rattachés aux rites liés à la vie familiale et collective, en tant que boisson partagée et offrande aux ancêtres.

D'une manière générale, les boissons alcoolisées sont consommées dans un cadre rituel, collectif et/ou festif et utilisées pour rendre hommage aux ancêtres, faire des offrandes aux multiples esprits, accompagner un sacrifice, etc. L'alcool est une substance rituelle d'importance, notamment là où intervient la transe (Le Failler 2000) afin de personnaliser et socialiser le contact médiumnique avec les entités invisibles. Si le whisky est la boisson qui marque un prestige social et une conduite ostentatoire du boire du fait de son prix élevé, le rhum, auquel est attachée la même symbolique qu'à l'hydromel, est actuellement la boisson de la transe par excellence.

Le rhum est appelé communément toaka mais, selon nos observations, il est désigné par le terme barisa quand le médium s'adresse aux ancêtres et quand il est en transe. Barisa est alors le nom donné au rhum par les esprits qui investissent le médium, ce qui indique la présence d'un vocabulaire spécifique à l'activité rituelle liée au boire religieux. La consommation d'alcool est ainsi contextualisée en fonction de pratiques religieuses ou ordinaires. Le mot barisa désignait plus précisément le miel mis en cuisson ou mélangé à de l'eau bouillante, ce qui le dote du pouvoir de faire naître la transe. Certains ethnologues dans d'autres régions de Madagascar (Estrade 1985 en région betsimisaraka), précisent que le barisa est l'hydromel ou la bière de canne à sucre. Pour d'autres (Berger 2006 en pays antankarana et Beaujard 2014 chez les Tañala), le barisa joue un rôle de purification et de mise en contact des vivants et des esprits. Les correspondances sont ici frappantes entre le rhum qu'apprécient les esprits et les autres substances connues sous ce même terme. Elles témoignent du lien symbolique entre fermentation alcoolique et décomposition des cadavres, comme « de la continuité des ancêtres et des esprits de la nature » (Beaujard 1995 : 580). L'idée de purification se retrouve également chez Nielssen lorsqu'elle parle des usages du barisa 
chez les Betsimisaraka. L'un de ses interlocuteurs dit à ce propos : « The barisa cleans the belly. When everybody drinks it before the bath, they all are cleansed, even those who have eaten taboo food » (2012: 181).

L'alcool caractérise certains types d'esprits, ancêtres familiaux bien sûr, mais aussi et surtout esprits royaux et vazimba (esprits chtoniens). En cela, ils se distinguent des autres esprits de la nature et plus précisément des ondines, comme les zazavavindrano, " filles-des-eaux », qui préfèrent les sodas, le miel, le lait et les bonbons, autant de boissons et de nourritures associées au féminin et exprimant la douceur du sucré, à l'opposé du caractère viril et guerrier prêté aux alcools. Cet usage rituel de l'alcool qui écarte les esprits aquatiques féminins, inscrit les autres esprits, buveurs de boissons fermentées (transformées socialement) ou de boissons « chaudes », dans un rapport de sociabilité avec les êtres humains.

Ces aspects du boire rituel qui associent et qui distinguent, permettent une identification collective, une lecture que Bianquis, parmi d'autres, développe à l'occasion de deux enquêtes menées dans des sociétés rurales, chez les viticulteurs alsaciens et les nomades de Mongolie. Au-delà de la sociabilité et de la communication avec la "surnature ", l'acte de boire de l'alcool renvoie aux rites d'identification collective. L'alcool apparaît comme un révélateur d'identité, une ligne de partage, de limite et de fracture (2012 : 12). De fait, les rituels de possession sont l'occasion de faire circuler le verre rempli de rhum. Si les participants aux rites n'ont pas l'obligation de consommer l'alcool proposé, tous acceptent d'en boire, ne serait-ce qu'une gorgée. A ce sujet, un devin-guérisseur raconte que ses vazimba sont généreux puisqu'ils partagent le rhum avec les personnes présentes au cours du rituel, le patient, son entourage et les familiers.

\section{Boire pour l'invisible}

Lors des rituels, l'ethnographe ne peut mesurer quantitativement le volume exact bu par le maître rituel, et ceci pour plusieurs raisons : bien souvent, le contenant est un gobelet en tôle émaillée, ce qui ne laisse pas voir le niveau rempli ; les absorptions d'alcool par le possédé peuvent être comptées en nombre de gestes mais pas en volume ingéré - trempe-t-il à peine ses lèvres ? boit-il une petite gorgée ou une pleine rasade ? ; le contenu des bouteilles n'indique pas plus le volume consommé puisqu'une part plus ou moins grande est réservée à l'ensemble des acteurs du rituel et destinée aux aspersions. Pour reprendre le questionnement de Nahoum-Grappe, « Mais qu'est-ce qu'un 'coup' ? Une gorgée ? Un verre ? Un geste ? » (1989: 72). Il faut ainsi retenir l'idée du geste dans l'expression « boire un coup » afin de mesurer le rythme rituel des absorptions plutôt que la quantité d'alcool absorbée.

L'ethnographie qui suit est issue d'un terrain situé dans la région rurale du nord-ouest de l'Imerina, sur les Hautes Terres centrales. Le style saccadé de la description permet de mettre en lumière ce qui nous intéresse ici, à savoir les usages du rhum durant une transe de possession. Ce jour-là, il s'agit d'une malade qui se plaint d'un mal de ventre. Elle est accompagnée de son mari et de sa mère. Cette dernière souhaite également s'entretenir avec le devin-guérisseur au sujet de problèmes relationnels. La séquence ethnographique est ainsi structurée en deux parties : la première concerne cette femme qui souffre du ventre, victime d'un acte de sorcellerie; la deuxième concerne la vieille dame qui dit être accusée par ses voisins d'être une sorcière. 
Le devin-guérisseur qui a diagnostiqué un acte de sorcellerie procède à un rituel pour extraire les aliments logés dans le ventre, aliments qui représentent l'acte malfaisant. Il est sous-entendu que l'assistant rituel remplit le gobelet du devin-guérisseur au fur et à mesure qu'il se vide. Une fois en transe, l'esprit qui possède le devin-guérisseur n'hésite pas à réclamer du rhum quand l'assistant manque à son service car il veut montrer qu'il est un buveur de rhum. Ainsi, il est en droit de réclamer, de demander, d'exiger. Il est à noter que tous les esprits qui possèdent ce devin-guérisseur sont des buveurs de rhum, même la défunte grand-mère Bebe ${ }^{2}$ Rajobo.

Dans le texte qui suit ne sont pas référencées toutes les fois où le verre est à nouveau rempli même si le geste est exécuté à plusieurs reprises. Les séquences où le rhum est utilisé sont présentées en alinéa afin que soient repérées plus facilement la fréquence et la nature des usages de l'alcool, parfois illustrées de captures d'écran qui apportent un éclairage sur les modalités rituelles du boire. Des indications temporelles sont également référencées pour que la temporalité rituelle soit mieux appréhendée. Cette séance filmée a une durée totale d'une heure et vingt-cinq minutes.

Une fois la patiente arrivée avec son mari et sa mère au domicile du devin-guérisseur, celui-ci prépare la petite natte en raphia appelée valamena ${ }^{3}$ qui circonscrit l'espace rituel où seront posés les objets sacrés. Elle est placée au coin nord-est de la pièce principale de la maison. Le mari de la patiente remet une petite bouteille de rhum appelée botakely ${ }^{4}$ au devin-guérisseur.

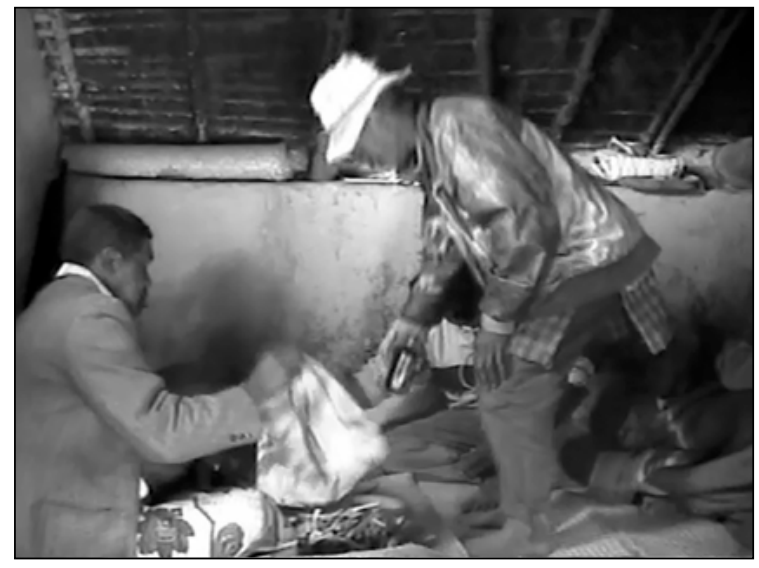

Figure 1: A la deuxième minute, le mari de la patiente, debout à droite, tend la petite bouteille de rhum au devin-guérisseur assis, à gauche de la photo. Il est en train de déballer ses outils rituels d'un sac - (C) Delphine Burguet, 2002

Le devin-guérisseur met son collier de perles astrologiques autour du cou et prend sa canne rituelle tandis que son assistant verse du rhum dans un gobelet en émail.

2 « Bebe » signifie grand-mère et vient du terme Nenibe.

3 «Enclos rouge » : couleur royale. Le terme désignait l'espace politique où se situait le roi ou son représentant.

4 «Petite bouteille » : contenance de $30 \mathrm{cl}$. 


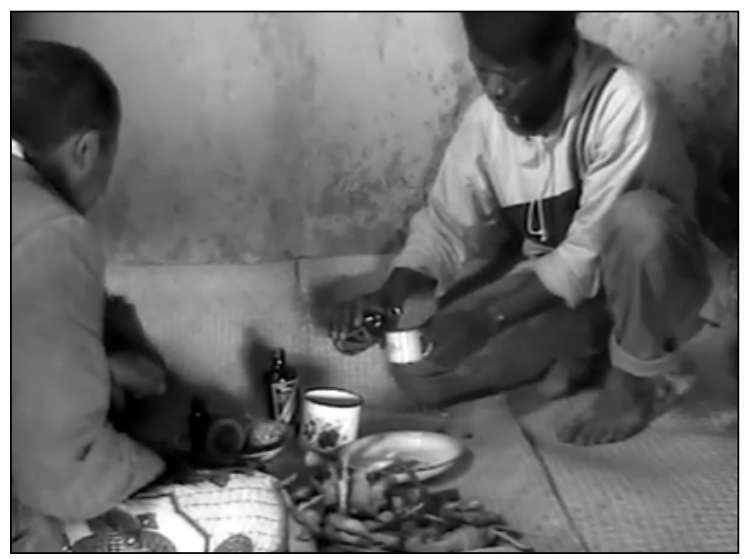

Figure 2 : A la cinquième minute, l'assistant rituel (à droite) verse du rhum dans un verre émaillé pendant que le devin-guérisseur sélectionne les bois sacrés à réduire en poudre - (C) Delphine Burguet, 2002

Puis il asperge de rhum le coin nord-est, destination zodiacale privilégiée pour invoquer les esprits.

Il appelle les esprits à l'aide de son miroir.

Il asperge le coin nord-est pour invoquer Bebe Rajobo, une ancêtre familiale qui vient posséder le devin-guérisseur lorsqu'il l'invoque dans un cadre rituel.

Le devin-guérisseur poursuit son invocation aux esprits.

Il asperge le coin nord-est à la fin de l'invocation.

Il noue un bandeau rouge, couleur royale, autour de sa tête et pose un carré de tissu rouge sur sa tête. Il se prépare pour l'entrée en transe.

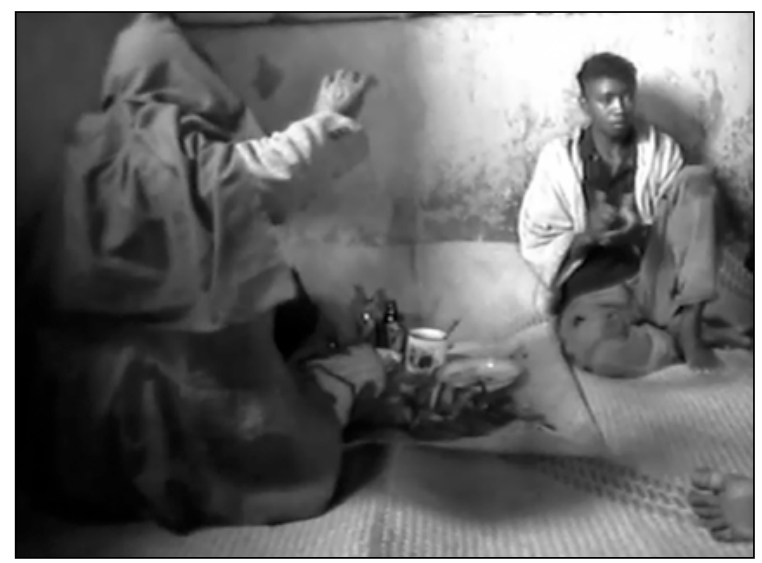

Figure3 : A la onzième minute, le devin-guérisseur, orienté en direction du Nord-est, souffle et balance son bras pour entrer en transe. Le carré de tissu rouge lui recouvre la tête. A droite, l'un de ses fils qui l'aident dans la préparation des remèdes à base de poudre de bois - C Delphine Burguet, 2002

Il souffle « tsh !! tsh !! » qui signifie l'entrée en transe et descend le carré de tissu rouge sur ses épaules. Le devin-guérisseur est à présent possédé par Bebe Rajobo. La veille femme salue tous les protagonistes : la patiente et ses proches, l'assistant rituel, les enfants, les voisins, les invités... 


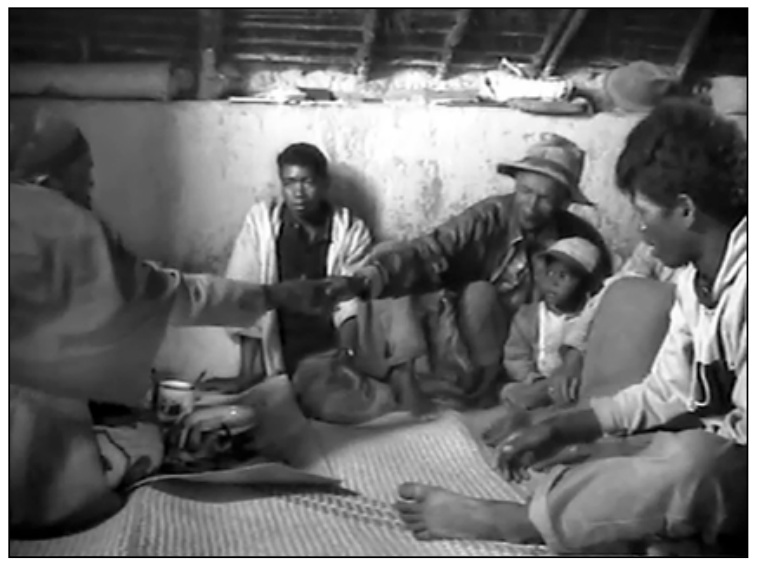

Figure 4 : A la dix-septième minute, le devin-guérisseur possédé salue de la main le mari de la patiente. C'est l'esprit qui le possède qui engage les salutations - C Delphine Burguet, 2002

Quelques paroles sont échangées autour des symptômes.

Le possédé asperge le coin nord-est.

Il boit, le premier, un coup de rhum contenu dans son gobelet (dans le discours émique, c'est Bebe Rajobo, l'esprit possesseur, qui boit).

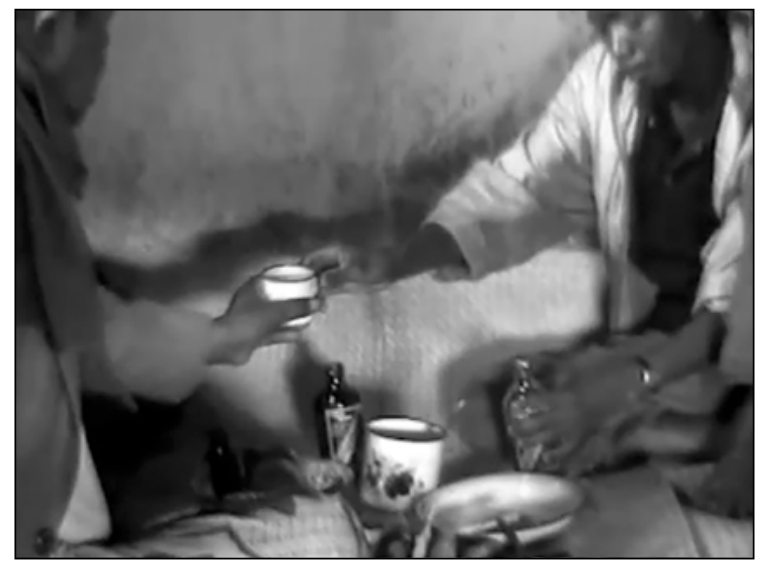

Figure 5 : A la dix-huitième minute, son assistant lui offre un verre de rhum - C Delphine Burguet, 2002

La patiente s'approche du possédé et enlève ses vêtements gênants pour la consultation. Elle ôte ainsi le haut de sa robe pour laisser apparaître son torse nu. Le devin-guérisseur a besoin d'un contact direct avec l'abdomen de la patiente pour réaliser le diagnostic puis les soins.

Le possédé verse du rhum dans deux bouteilles qui contiennent des remèdes sous forme liquide, qu'il appelle des fahasivy.

Le possédé utilise son miroir pour vérifier le diagnostic. Il l'applique sur le ventre de la patiente pour confirmer que le mal est bien logé à cet endroit précis. Puis le pose successivement sur la nuque et le dos. En fonction de ce nouveau diagnostic, il prépare 
les charmes thérapeutiques à base de bois sacrés. Il y ajoute de la poudre de coquillage, de la poudre de la porte d'entrée en bois de la pièce principale de son domicile et de son charme de protection appelé mohara.

Il mélange à nouveau du rhum dans les deux bouteilles qui contiennent les fahasivy. Il boit un coup.

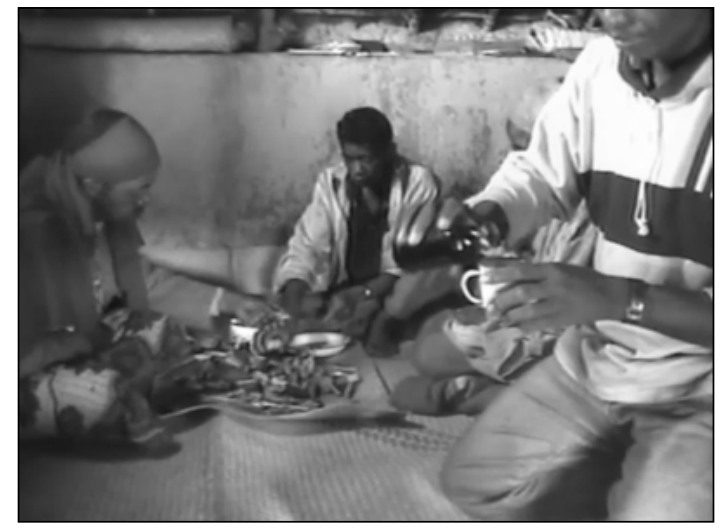

Figure 6 : A la vingt-et-unième minute, l'un de ses assistants au premier plan lui sert un verre de rhum tandis que le plus jeune se charge de réduire les bois sacrés en poudre - $\odot$ Delphine Burguet, 2002

Le possédé procède au rituel thérapeutique en utilisant sept allumettes, un van en osier utilisé pour nettoyer les grains de riz et un balai qu'il fait tourner sept fois autour de la patiente. Puis, elle doit inhaler la fumée d'un charbon ardent qui permet de chasser le mal logé dans le ventre. Le possédé frappe la patiente à l'aide d'un tissu pour faire partir définitivement l'esprit nuisible.

Le possédé fait boire à sa patiente le remède contenant le rhum.

Il boit à même la bouteille de rhum.

Le possédé verse du rhum sur une lame de rasoir.

A l'aide de cette lame de rasoir arrosée de rhum, il procède à une incision de la peau au niveau du ventre de sa patiente, à l'endroit où le miroir avait été posé précédemment.

Il boit un coup.

Il se rapproche de l'incision et aspire pour en recracher des morceaux de poulet.

Il verse du rhum sur l'incision une fois l'extraction terminée.

Il boit un coup.

Le possédé fait boire à sa patiente deux gorgées de remède contenant du rhum.

A nouveau, le possédé procède à une incision du côté droit du ventre de sa patiente. Il incise, aspire et recrache du sang.

Il asperge le coin nord-est.

Il demande à un autre « soldat», autre esprit qui combat le mal, de venir pour agir.

Le possédé fait boire à sa patiente deux gorgées de remède contenant du rhum.

Le possédé aspire à nouveau et crache des morceaux de manioc.

Il verse du rhum sur l'incision. 
"Les ancêtres aiment boire du rhum!" (In)visible ivresse et questions réflexives sur le boire rituel

Il verse du rhum dans les deux bouteilles contenant les remèdes et fait boire une gorgée de l'une d'entre elles à sa patiente.

Le mari de la patiente va acheter une petite bouteille de rhum.

Le possédé fait boire à sa patiente une gorgée de remède mélangé au rhum.

Quand le mari de la patiente revient avec le rhum, le possédé boit un coup, à même la bouteille.

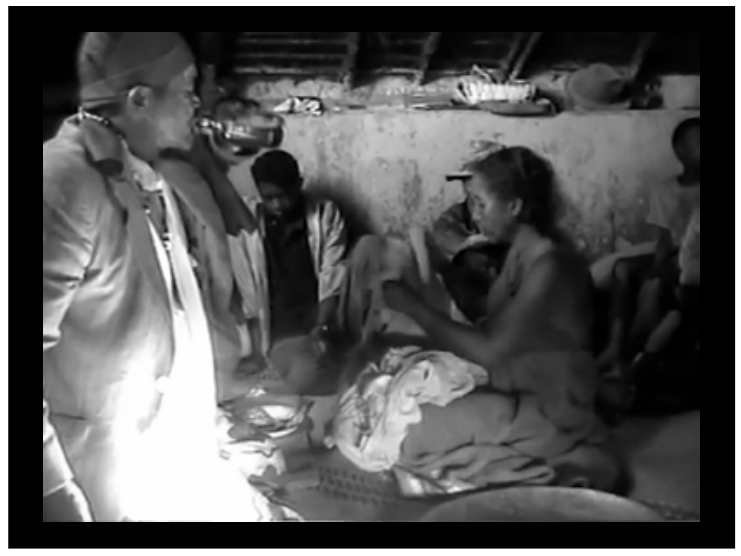

Figure 7 : A la cinquantième minute, la patiente se rhabille, la consultation étant terminée. Le possédé boit un coup à la bouteille - ( C) Delphine Burguet, 2002

Le possédé remet son carré de tissu rouge sur la tête pour signifier la sortie de transe mais la séance n'est pas terminée, un autre esprit vient le posséder. Une discussion est engagée avec le mari puis la mère de la patiente.

Il boit un coup.

La vieille femme explique qu'elle est sujette à des moqueries. Les jeunes de son village la considèrent comme une sorcière. L'esprit qui possède le devin-guérisseur procède alors à un rituel de protection.

Il asperge son miroir de rhum.

Il regarde son miroir pour deviner et considère qu'il n'y pas de problèmes du côté de la grand-mère. En fait, l'esprit veut vérifier l'honnêteté de la grand-mère.

Il boit un coup. 


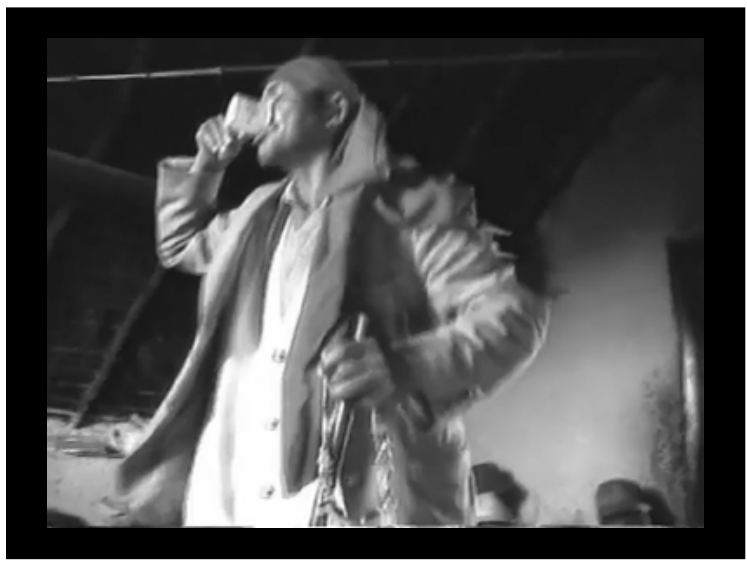

Figure 8 : A la cinquante-troisième minute, le devin-guérisseur possédé, debout, tenant de sa main gauche son collier de perles astrologiques et son bâton rituel, boit un coup - ( ) Delphine Burguet, 2002

Il confectionne un charme de protection à base de poudre de bois sacrés qu'il mélange à de l'eau.

Il rajoute du rhum dans le charme.

Il fait boire à la grand-mère sept gorgées du remède liquide fahasivy contenant du rhum.

Il verse un peu de remède contenant du rhum sur la tête de la grand-mère.

Le possédé boit un dernier coup.

La grand-mère inhale la fumée d'une composition de plantes préparées à cette occasion. Puis, le possédé pose son carré de tissu rouge sur la tête pour signifier le départ de l'esprit possesseur. Il salue les protagonistes pour dire au revoir et se positionne assis en direction du nord-est. Il bouge, s'agite et souffle « tsh !! tsh !! ».

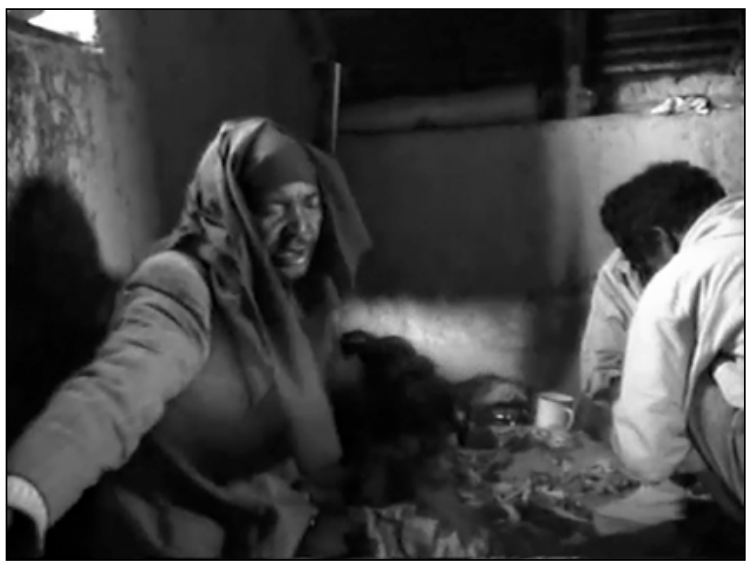

Figure 9 : Après une heure et vingt minutes, le devin-guérisseur a placé le carré de tissu rouge sur sa tête pour effectuer la sortie de transe. Il bouge le bras droit de manière circulaire et tient son bâton rituel dans la main gauche. A droite, les deux assistants rituels terminent de réduire en poudre les végétaux - (C) Delphine Burguet, 2002 
A peine une minute plus tard, l'esprit quitte le devin-guérisseur. Celui-ci se déplace légèrement pour s'adosser au mur.

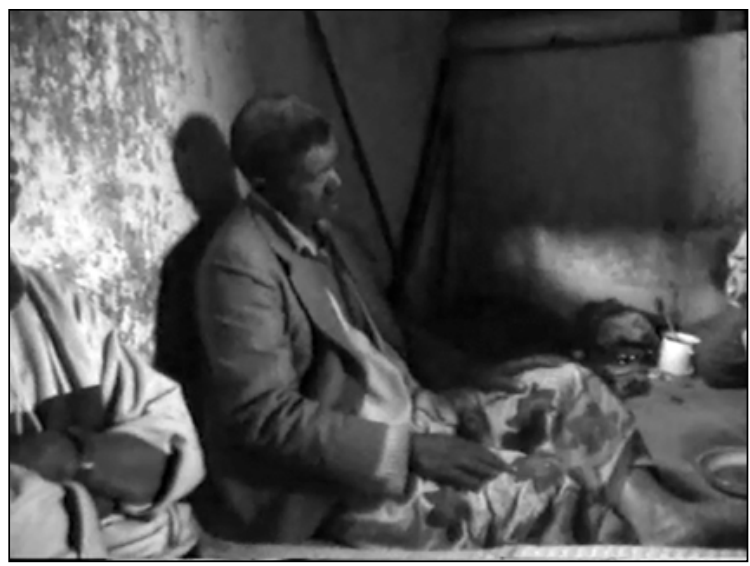

Figure 10 : Le devin-guérisseur est adossé au mur, jambes croisées. Il semble fatigué, la tête penchée sur le côté, sans mot dire - (C) Delphine Burguet, 2002

Cette étude de cas ethnographique montre les différents usages du rhum et du boire rituels : aspergé, le rhum montre l'attention portée au monde de la surnature à l'occasion des invocations ; mélangé aux remèdes, il valorise symboliquement les substances bénéfiques et thérapeutiques qui, sous forme liquide, sont elles-mêmes aspergées et avalées ; versé sur le miroir, il active la clairvoyance et les facultés de deviner ; appliqué, il désinfecte les objets et les plaies; bu, il humanise et socialise les esprits.

L'ethnographie porte son regard sur le boire alcoolisé et non pas sur les boissons, une différence lexicale qui suppose une analyse des pratiques sociales et culturelles signifiantes du boire : « le 'boire' recouvre tout autant la substance elle-même (la boisson) que les pratiques sociales et significations culturelles qui entourent sa consommation et qui confèrent au 'boire' alcoolisé (ici, au boire) son originalité » (Obadia 2014 : 2). Cet angle d'analyse propose de considérer l'usage de l'alcool comme la figuration de valeurs sociales et culturelles positives d'un groupe (ibid.). Le rhum que boivent les ancêtres fait figure de valeur religieuse et de ciment social tant que le médium maîtrise la transe et dissimule les signes visibles de l'ivresse.

\section{Visionner l'ivresse}

Le problème de la définition de l'ivresse (Fabre-Vassas 1989) se pose ici, appréhendée en fonction de codes sociaux variables en lien au « boire ». Ce questionnement fait suite aux analyses proposées par Mary Douglas (1987) et Claudine Fabre-Vassas (1989) qui ont développé l'idée qu'une efficacité symbolique de la consommation d'alcool aurait un effet pathologique moindre. Ainsi, le contexte rituel amènerait une gestion spécifique des signes de l'ivresse. Non seulement la consommation de rhum est un outil de mesure de compétence dans le champ rituel - le devin-guérisseur qui ne montre pas les signes d'ivresse est considéré comme un maître confirmé - mais elle est également un outil de mesure de la gestion du corps et de ses limites. La transe invite le dépassement de soi pour celui qui met en scène les ancêtres, dépassement qui s'accompagne d'expériences 
des limites du corps au boire. L'enjeu social de la gestion du boire est celui de la maîtrise dans le jeu des limites rituelles sans cesse repoussées à l'aide de l'alcool. Un jeu de bascule s'entrevoit entre une mesure et une démesure, qui crée la maîtrise et justifie une position sociale. Bien entendu, dire que les devins-guérisseurs ne sont pas ivres revient à dire qu'ils arrivent malgré le boire à garder une maîtrise de soi, ce qui donne à voir une domination sur l'alcool, et ainsi une capacité à accueillir de puissants esprits.

Sur ce terrain, il n'est pas question de boire jusqu'à tomber « ivre-mort », ou relever le défi de boire avec d'autres, jusqu'à perdre la face, ni de participer à une ivresse collective ou festive au nom des ancêtres. Il est vrai que dans bien des sociétés et des espaces de sociabilité, l'alcool est un partage commensal ou un jeu de défi masculin. Ce terrain offre, à l'inverse, une autre façon de boire où l'ivresse invisible, indécelable, prévaut. C'est là tout l'art de la maîtrise de soi ou de la maîtrise du corps dans l'exercice de la transe de possession.

Lors de l'engagement rituel, il semble que cette invisible ivresse soit renforcée par l'usage de la caméra, considérée comme une focale, un point de vue particulier, où l'on capte sans prendre en considération l'ensemble de l'intentionnalité et de l'effervescence des pratiques. Contrairement à l'œil nu, son champ de vision réduit parfois la vue d'ensemble. On peut également se demander si l'objet, qui fait l'interface entre l'œil et les personnes filmées, n'ajoute pas un filtre au champ de vision. Agent médiateur et objet déformant, la caméra amène à interroger la performance des conduites d'alcoolisation et de la transe codifiée.

Alors que le regard est déformé par le filtre de la caméra, il devient plus clair au moment du visionnage des images. Plusieurs ethnologues défendent cet aspect méthodologique d'une approche visuelle du terrain d'enquête comme une valeur ajoutée. En lien au boire alcoolisé et dans un contexte de transe de possession, la prise d'images révèle de multiples facettes sociales au niveau des conduites et des interactions. Mead et Bateson (1977) questionnaient déjà l'usage de la caméra et de la photographie en anthropologie : les deux outils étaient parfois utilisés lors d'une même séance d'observation sur le terrain d'enquête : la caméra sur un trépied, l'appareil photo autour du cou. Comme chez Barthes (1980), Dubois (1983), Colleyn (1988) ou encore Piette (1998, 2007), l'usage réflexif des images prises sur le terrain amène une distance où celles-ci sont révélatrices de ce qui est là sans avoir été vu, notamment au cours de l'action observée. Le film amène une description des rituels quasiment continue et garantit l'authenticité des objets, tout comme des aspects peu décelables des conduites sociales (Burguet 2017). Ainsi, l'image selon Piette (2007 : 26) renseigne sur « la position interpersonnelle, les mouvements interpersonnels, l'intensité de l'engagement, les positions du corps, l'intensité des mouvements gestuels de chaque partie du corps, les types de regard, les zones de déplacement du corps pour effectuer un mouvement déterminé ».

L'expérience ethnographique questionne l'ivresse qui, contrairement aux discours des buveurs, est décelable lorsqu'ils boivent « un peu trop », « trop rapidement » ou encore « outre mesure » durant le rituel. C'est la prise de distance d'avec le déroulé rituel et le terrain d'enquête qui donne l'ivresse, la même qui semblait indécelable auparavant, dans l'action rituelle. Si, dans un premier temps, le rhum paraît donner de la vigueur au médium, des troubles physiques et psychiques arrivent. Le corps se déséquilibre. Des gestes sont manqués. La mémoire fait défaut. L'élocution se fait difficile. Les 
muscles du visage se relâchent. Dans toutes les situations et encore plus dans celles-ci, les fidèles détournent leurs visages et baissent leurs regards, car pour eux, le maître rituel fait figure d'autorité (Burguet 2014, 2017). Il n'est alors pas question de rendre visible les émotions. Si l'on reprend les captures d'écran qui illustrent l'étude de cas ethnographique présentée plus haut, on voit que le devin-guérisseur ne termine pas sa séance de possession comme il l'a commencée. Même si ces images ne sont pas aussi démonstratives que celles successives qui forment le film, on comprend que le devinguérisseur subit à la fois la fatigue de la performance de transe et de l'ivresse.

Après le premier temps où l'on filme et le deuxième où l'on visionne le film pour la première fois, arrive le troisième temps de l'analyse qui est celui du visionnage critique de ce qui a été capté ou capturé. Les signes visibles d'ivresse apparaissent alors sur les images, que l'on découvre « après coup »; c'est ainsi que se dessine une autre vision de l'activité rituelle en lien au boire alcoolisé.

Quand on côtoie le devin-guérisseur dans son quotidien (il est éleveur de zébus et agriculteur) et quand on l'observe dans un contexte magico-religieux, lorsqu'il est possédé par les esprits, on se rend compte de l'évolution des changements physiques et psychiques provoqués par l'ivresse. Ils se lisent sur le visage du possédé qui, dans le bal des masques, porte celui de l'homme ivre. Ils s'accompagnent d'autre signes qui entrent dans le rituel, comme fumer une cigarette, pratique qu'il ne fait pas dans un espace-temps ordinaire, et danser sur le rythme saccadé d'une musique de transe jouée par l'accordéoniste.
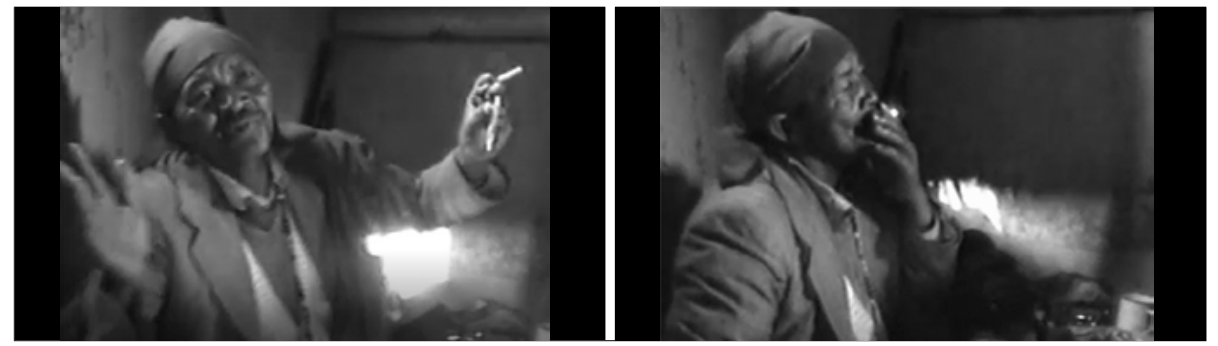

Figures 11 et 12 : Le devin-guérisseur possédé par un esprit vazimba fume une cigarette. Il met en scène l'esprit qui aime fumer - (C) Delphine Burguet, 2002
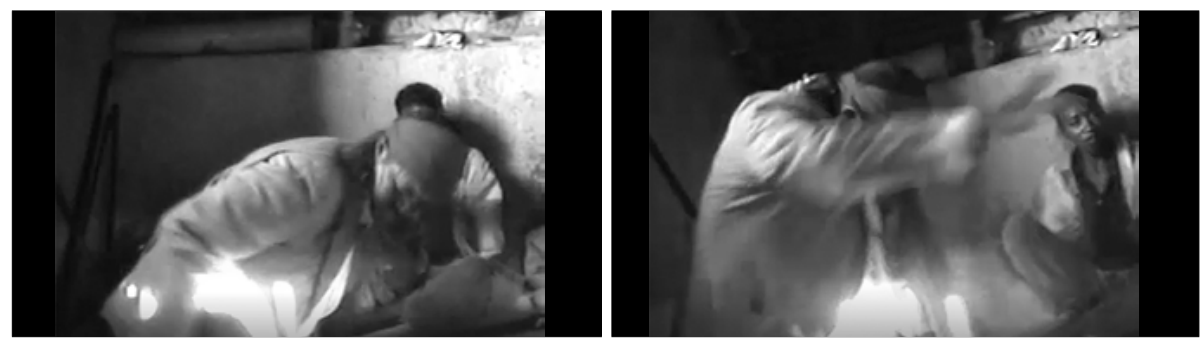

Figures 13 et 14 : Le devin-guérisseur, en transe, danse au rythme de l'accordéon - C Delphine Burguet, 2002 


\section{Taire l'ivresse}

Après ce premier temps d'analyse qui consiste à visionner les films, cet aspect visible « après coup » de l'ivresse rituelle a été mis aux marges de l'enquête, tenu à distance. L'ivresse et la démesure qui s'opposent au contrôle et à la codification des rituels ne doivent pas être visibles lors des transes de possession puisque, selon la logique émique, ce sont les ancêtres qui boivent le rhum. La question réflexive montre ainsi le point de vue différencié entre un discours émique et une analyse distanciée. Car en fait, l'action des enquêtés présente une réalité, la leur, qui oriente le regard de l'enquêteur. Ceci amène à réfléchir sur la hiérarchie de ce qui importe aux yeux des différents acteurs et sur la capacité de ces derniers à imposer cette hiérarchie aux autres, y compris à l'ethnologue.

Au sujet de ce boire ritualisé, le questionnement est apparu bien après les différentes phases d'enquête de terrain. L'acceptation de l'idée que ces maîtres étaient en état d'ivresse lors des séances de possession n'a été possible que dans un espace-temps situé en dehors des interactions et des expériences vécues collectivement. Ce décalage dans l'acceptation s'explique par le fait d'écouter l'interprétation émique des faits de possession où le discours, comme nous l'avons vu, valorise l'absence d'ivresse. Le discours est énoncé pour convaincre celui qui écoute que ce qui est dit est vrai. Bien entendu, au-delà de la parole discursive, l'observation amène à « vérifier » ou à voir ce qui se passe dans l'action rituelle.

Parler d'ivresse dans ce contexte suppose la remise en question du discours et la déconstruction de la représentation méliorative du buveur. Longtemps écartées, les données ethnographiques sur l'ivresse ont été exploitées bien après le temps du terrain ${ }^{5}$. Elles marquent une étape importante dans cette démarche réflexive : l'ivresse rituelle, comme objet d'étude, a été réintégrée dans la logique d'analyse d'une anthropologie du boire et dans un rapport distancié au terrain ethnographique. Cet écart dans le traitement des données empiriques questionne ce sentiment de trahison. Si l'ivresse est perçue comme un sujet sensible pour les devins-guérisseurs, l'ethnologue qui analyse cette ivresse visible a l'impression de faire tomber les masques du jeu social de ceux qui incarnent les puissances invisibles. Faire tomber les masques chez les possédés et devins-guérisseurs confirmés, c'est révéler non seulement une possible faiblesse de ces esprits (d'un point de vue émique) mais aussi du rôle social que jouent ces médiateurs avec le monde de la surnature.

\section{Conclusion}

Les expériences liées au boire alcoolisé sur ce terrain ne concernent pas l'ethnologue mais celles du maître rituel car les conditions économiques et les codes religieux limitent l'usage de l'alcool pour les participants, en dehors du médium. Outre la commensalité dictée par les esprits buveurs de rhum qui nous invitent à prendre une gorgée, il n'est pas envisagé que les participants en boivent davantage puisque la boisson leur est réservée. La logique sociale de l'activité rituelle fait que seul le possédé se situe en conduite d'alcoolisation. Il se distingue par-là des autres individus de sa communauté,

5 A ce sujet, un épisode d'ivresse intense lors d'un rituel de soins a fait l'objet d'une publication (Burguet 2016). 
non détenteurs de la maîtrise de la transe de possession. Ainsi, j'ai été invitée à boire tout comme les autres protagonistes mais sans jamais dépasser la mesure imposée. La réflexivité évoque plutôt l'expérience du boire du devin-guérisseur et la façon d'appréhender les conduites d'alcoolisation qui en découlent, en fonction du regard que l'on lui porte.

Ecrire sur l'ivresse, c'est remettre en question le discours que les médiums tiennent sur leur capacité physique et psychique, et bien entendu sur l'authenticité de la transe qu'ils mettent en scène à chaque rituel. Le niveau d'analyse n'est alors pas le même entre décrire un rituel où le possédé fait régulièrement le geste de boire du rhum et décrire la visibilité de l'ivresse, si l'ivresse est perçue comme une défaillance pour les acteurs du rite. La quête d'une analyse réflexive proposée par le prisme du boire alcoolisé permet de valoriser l'arrière-scène, auparavant voilée, des expériences de l'enquête de terrain et les façons de faire de la production scientifique. Petit (2010) écrit que « Nul ne parle de nulle part, et l'on est en droit d'attendre que l'ethnographe rende avec une solide dose de transparence les chemins souvent tortueux qui l'ont amené à développer sa réflexion dans telle direction plutôt que dans telle autre ». En filigrane, il est question d'informations hiérarchisées, classées et interprétées en fonction d'un lien relationnel au terrain qui s'associe à l'affect et à la longue durée, et selon l'orientation donnée au regard de l'ethnographe par les enquêtés sur leur propre réalité. Comme on l'a vu, l'agencement des données recueillies sur le terrain est une construction à la fois scientifique et implicite qui influence la production de l'analyse.

L'ethnologue hiérarchise ses données selon des intérêts scientifiques bâtis en fonction d'hypothèses mais aussi en rapport avec son expérience propre du terrain d'enquête. Par conséquent, les données empiriques qui risquaient de faire tomber les masques ont été mises de côté, repoussées, refoulées. Cette hiérarchisation des données qui amène à nier et à oublier ce que l'on a vu rappelle ce que Patricia et Peter Adler (2000) expliquent par l'idée d'autocensure par loyauté ou par l'angoisse de trahison.

Dans un premier temps, je n'ai pas vu l'ivresse, prise dans l'action rituelle, l'engagement social et située derrière la caméra ; par la suite, focalisée sur l'analyse des films, j'ai vu cette ivresse à l'écran, mais sans pour autant en rendre compte. C'est dans un dernier temps que j'ai finalement proposé une lecture de l'état d'ivresse lié au boire religieux. La progression réflexive montre que le processus d'autonomisation vis-à-vis des personnes étudiées évolue en fonction des enjeux que l'écriture analytique soulève. Cette autonomisation suppose de considérer un temps parfois long, d'autant plus long que le terrain a été approfondi et dense. Parmi les notes et souvenirs de terrain, une dissociation s'effectue quant à la nature des évènements et des conduites observés qui rend compte d'une classification des informations et des expériences ethnographiques. L'ivresse ritualisée n'y échappe pas. 


\section{Références citées}

Adler, Peter et Patricia A. AdLer, 2000. «L'autocensure dans les sujets sensibles », in Jacqueline Feldman, et Ruth CANTER KoHN (dir), L'éthique dans la pratique des sciences humaines : dilemmes, pp. 163180. Paris : L'Harmattan.

BARTHES, Rolland, 1980. La chambre claire. Paris : Gallimard, Le Seuil.

Bateson, Gregory et Margaret MeAD, 1977. "On the Use of the Camera in Anthropology", Studies in the Anthropology of Visual Communication, 12, pp. 78-80.

Beaujard, Philippe, 1995. "Religion et société à Madagascar. L'exemple tañala ", L'étranger intime. Mélanges offerts à Paul Ottino, pp. 181-217. Université de la Réunion : Océan Editions.

Beaujard, Philippe, 2014. « Mythe et rituels. Le miel et l'hydromel dans quelques sociétés de Madagascar », Etudes Océan Indien, $\mathrm{n}^{\circ}$ 51-52, pp. 129-169.

BERGER, Laurent, 2006. Les raisons de la colère des ancêtres zafinifotsy. Le discours anthropologique à l'épreuve de la mondialisation. Thèse de doctorat. EHESS, 820 p.

BIANQUis, Isabelle, 2012. L'alcool. Anthropologie d'un objet-frontière. Paris : L'Harmattan.

BuRguet, Delphine, 2014. Figures des maîtres rituels. Les devins-guérisseurs dans l'histoire et aujourd'hui. Savoir, action et pouvoir à Madagascar. Thèse de doctorat, EHESS, $530 \mathrm{p}$.

Burguet, Delphine, 2016. « La transe de possession chez un devin-guérisseur confirmé (Vonizongo, Nordouest de l'Imerina, Madagascar) », in Sébastien BAUD (dir.), Anthropologies du corps en transes, pp. 135-156. Paris : Editions Connaissances et savoirs.

Burguet, Delphine, 2017. « Un rituel de soins au bord de l'eau et les images de l'autorité thérapeutique », Images du travail, Travail des images, 4 - Mis en ligne le 20 juin 2017, consulté le 23 mai 2018 $<$ http://imagesdutravail.edel.univ-poitiers.fr/index.php?id=1413>

Colleyn, Jean-Pierre, 1988. « Anthropologie visuelle et études africaines », Cahiers d'études africaines, 111-112, pp. 513-526.

Douglas, Mary, 1987. Constructive drinking. Perspectives on drink from anthropology. Cambridge University Press. Paris: Maison des sciences de l'homme.

DuboIs, Philippe, 1983. L'acte photographique. Paris : Nathan.

Estrade, Jean-Marie, 1985. Un culte de possession à Madagascar : le tromba. Paris : L'Harmattan.

FABRE-VASSAS, Claudine, 1989. « La boisson des ethnologues », Terrain, 13, pp. 5-14.

FAHRASMANE, Louis et Berthe GANOU-PARFAIT, 1997. De la canne au rhum. Paris : Editions de l'INRA

Fremigacci, Jean, 2014. Etat, économie et société coloniale à Madagascar (fin 19e siècle-1940). Paris : Karthala.

Feeley-HaRniK, Gillian, 1991. A Green Estate. Restoring Independence of Madagascar. Washington et Londres : Smithonian.

Huetz De Lemps, Alain, 1997. Histoire du rhum. Paris : Editions Desjonqueres.

LAmbeK, Mickael, 1981. Human Spirits: A Cultural Account of Trance in Mayotte. Cambridge : Cambridge University Press.

Le FAILler, Philippe, 2000. Opiums : les plantes du plaisir et de la convivialité en Asie. Paris : L'Harmattan. Nahoum-Grappe, Véronique, 1989. « Boire un coup... », Terrain, n 13, pp. 72-80.

NielsSEn, Hilde, 2012. Ritual Imagination. A Study of Tromba Possession among the Betsimisaraka in Eastern Madagascar. Boston : Brill.

OBADIA, Lionel, 2004. « Le 'boire'. Une anthropologie en quête d'objet, un objet en quête d'anthropologie », Socio-anthropologie, 15 [En ligne], <http://socio-anthropologie.revues.org/421>

Petit, Pierre, 2010. « L'ethnographieur ethnographié », Dits, 14, pp. 12-23.

PIETTE, Albert, 1988. « Les détails de l'action », Enquête, EHESS, pp. 109-128.

PIETTE, Albert, 2007. « Fondements épistémologiques de la photographie », Ethnologie française, 37 (1), pp. 23-28. 